\title{
Research on Correlation Between Morphological Changes in Ankle Mortise and Ankle Joint Functions After a Pilon Fracture Surgery
}

\author{
Jinshan Wang, Qiang Li, Qiang Zhang, Yunfei Wang* \\ Department of Osteology, Inner Mongolia Bayannaoer City Hospital, Linhe, Inner Mongolia Autonomous Region, China \\ Email address: \\ 39222646@qq.com (Yunfei Wang) \\ ${ }^{*}$ Corresponding author
}

\section{To cite this article:}

Jinshan Wang, Qiang Li, Qiang Zhang, Yunfei Wang. Research on Correlation Between Morphological Changes in Ankle Mortise and Ankle Joint Functions After a Pilon Fracture Surgery. Journal of Surgery. Vol. 4, No. 3, 2016, pp. 65-70. doi: 10.11648/j.js.20160403.11

Received: June 6, 2016; Accepted: June 27, 2016; Published: June 30, 2016

\begin{abstract}
To discuss the correlation between morphological changes in ankle mortise and ankle joint functions after a surgery for Ruedi-Allgower Type III Pilon fracture. A retrospective analysis was conducted for 60 patients who received operative treatment for Ruedi-Allgower Type III pilon fracture and attended a follow-up visit from January 2007 to December 2012, among which, 32 were male and 28 were female; aged 20-25 years and averaged at 38 years; the time for follow-up visit is at 18-24 months averaged at 20 months. On the X-ray film photographed at the last follow-up visit, the following indexes were measured: (I) Height of ankle mortise, (2) Width of ankle mortise, (3) Depth of ankle mortise, (4) Angle of ankle mortise coronal, (5) Angle of ankle mortise sagittal view The functions of ankle joint on the affected side were scored and grouped at the last follow-up visit based on such scoring methods as Mazur and the like. 41 cases were assigned to the Excellent Group, 12 cases were assigned to the Good Group and 7 cases were assigned to the Moderate+Poor Group. The correlation between morphological changes in the ankle mortise and functions was analysed based on comparison among the five indexes of the ankle mortises on the affected side and the unaffected side of 60 patients and comparison of the difference values of the indexes for the affected side and unaffected side among the three groups. The research has shown that all differences in width of ankle mortise, depth of ankle mortise, coronal view and sagittal view angles have statistical significance except that height of ankle mortise does not have statistical significance based on the comparison among various indexes for the affected sides and the unaffected sides of 60 patients. The remaining four difference values exhibit negative correlation with scores and difference values increase with decreasing scores. It indicates that treatment of ankle mortise width, depth, angles of coronal view and sagittal view during operation exhibits significant correlation with postoperative ankle joint functions. Anatomical reduction of ankle mortise width, depth, angles of coronal view and sagittal view for patients with Ruedi-Allgower Type III pilon fracture during operation imposes a significant effect on postoperative functions of ankle joints. Thus the anatomic form of ankle mortise should be recovered as much as possible.
\end{abstract}

Keywords: Ankle Joint, Fracture, Anatomy, Therapeutic Outcome

\section{Introduction}

The ankle joint is largest flectional joint of human body consisting of extremitas inferior tibiofibula and trochlea of talus and articular facet medial malleolus and articular facet of lateral malleolus constitute the ankle mortise. Morphological changes in ankle mortise can reduce the contact area between the tibial spur and the joint, unbalance the bearing pressure, disturb the load transmission and cause traumatic arthritis finally [1-2]. Clinically, pilon fracture is a common fracture and the fundamental purpose of its operative treatment is to obtain a stable and painless joint with motion function. The operation should recover smoothness of the tibial spur articular surface as much as possible. Cutaneous necrosis, acra-hemodynamic disorder and traumatic arthritis should be prevented during treatment [4]. Whether the reconstructed ankle mortise profile is complete and articular surface is smooth not only affect ankle joint motion but also serve as 
important indexes for prediction of traumatic arthritis [5].

The research aims to discuss the correlation between morphological changes in the ankle joint on the affected side and function scores after a surgery for the Ruedi-Allgower Type III pilon fracture, analyse the effect of morphological changes in the ankle joint on the ankle joint functions, causes of ankle mortise changes and essentials for ankle mortise reconstruction by surgery.

\section{Material and Methods}

\subsection{General Data}

A retrospective analysis was conducted for 60 patients who received operative treatment for Ruedi-Allgower Type III pilon fracture and attended a follow-up visit from January 2007 to December 2012, among which, 32 were male and 28 were female; aged 20-25 years and averaged at 38 years. Causes of injury: 17 cases due to traffic accident and 34 cases due to high-altitude falling. The time between injury and surgery was $12 \mathrm{~h}-7 \mathrm{~d}$ averaged at $3 \mathrm{~d}$. All patients suffered from a fresh fracture, among which 8 patients were diagnosed as open fracture ( 5 cases were of Gustilo Type I and 3 cases were of Gustilo Type II) and 52 patients were diagnosed as closed fracture. The time for follow-up visit was between 18 and 24 months averaged at 20 months.

The functions of ankle joint on the affected side were scored and grouped at the last follow-up visit based on such scoring methods as Mazur and the like. 41 cases were assigned to the Excellent Group (90-100 points), 12 cases were assigned to the Good Group (80-90 points) and 7 cases were assigned to the Moderate+Poor Group (80 points).

\subsection{Therapeutic Method}

Among the 60 patients in the group, 14 patients underwent an emergency operation within $12 \mathrm{~h}$ with 8 patients diagnosed as open fracture; 37 patients received operative treatment within $3 \mathrm{~d}$; 9 patients underwent a surgery within $7 \mathrm{~d}$.

Based on displacement fracture and degree of injury of the surrounding soft tissues, three operative methods were adopted respectively.

(I) open reduction \& internal fixation operation for fracture: 35 patients, among which 5 patients were diagnosed as open fracture (Gustilo Type I). All operations were performed in in accordance with the AO principle

(II) limited internal fixation in combination with external fixator for treatment: 19 patients, among which 2 patients were diagnosed as open fracture (Gustilo Type II). The universal joint at the distal end of support was loosened to allow for motion of ankle joint 4-6 weeks after operation. Dismantle the external fixator 3-6 months after operation if the X-ray film showed signs of fracture healing.

(III) Stepwise deferred open reduction \& internal fixation for treatment: 6 patients, among which 1 patient was diagnosed as open fracture (Gustilo Type II). The distal tibia was treated with open reduction and internal fixation after temporary support fixation or calcaneal traction and detumescence symptomatic treatment for $7-14 \mathrm{~d}$ and regression of soft tissue swelling. Non-weight bearing exercise of active and passive flexion and extension of ankle joints was performed 4-6 weeks after operation [6].

\subsection{Indexes for Postoperative Follow-up Observation}

Ankle joint mortise and lateral side were radiographed at postoperative 3, 6, and 12-month and the last follow-up visit and the following indexes were to be measured.

(I) Height of ankle mortise: a perpendicular line was constructed to the medial axis of the tibia through the tip of lateral malleolus, the distance between the intersection point and the intersection point of the medial axis of the tibia and articular surface of the tibia as shown on the X-ray film for ankle mortise (Figure 1).

(II) Width of ankle mortise: the distance between the connected line of two fornix apexes of the astragalus and the two points of intersection with medial and lateral malleolus as shown on the X-ray film for ankle mortise (Figure 2).

(III) Depth of ankle mortise: the distance between the anterior and posterior malleolus rims as shown on the lateral projection X-ray film for ankle joint (Figure 3).

(IV) coronal view angle of ankle mortise (a): angle made between the medial axis of the tibia and the perpendicular line to two the connected line of the fornix apexes of the ankle mortise as shown on the X-ray film for coronal view (Figure 4).

(V) sagittal view angle of ankle mortise $(\beta)$ : angle made between the medial axis of the tibia and the perpendicular line to two the connected line of the anterior and posterior malleolus rims as shown on the lateral projection X-ray film (Figure 5).

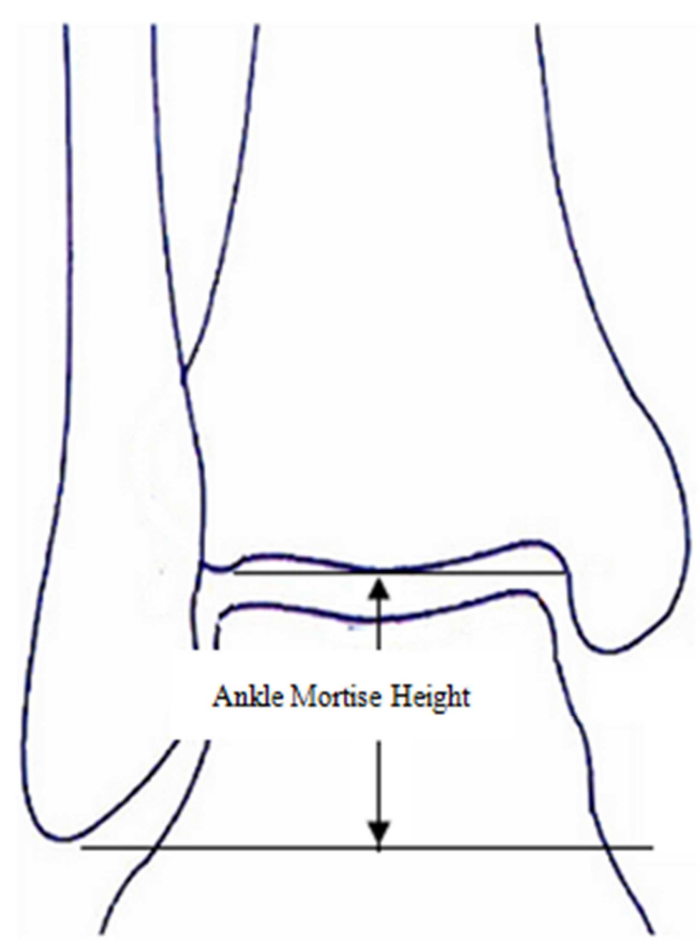

Figure 1. Schematic Diagram for Height of the Ankle Mortise. 


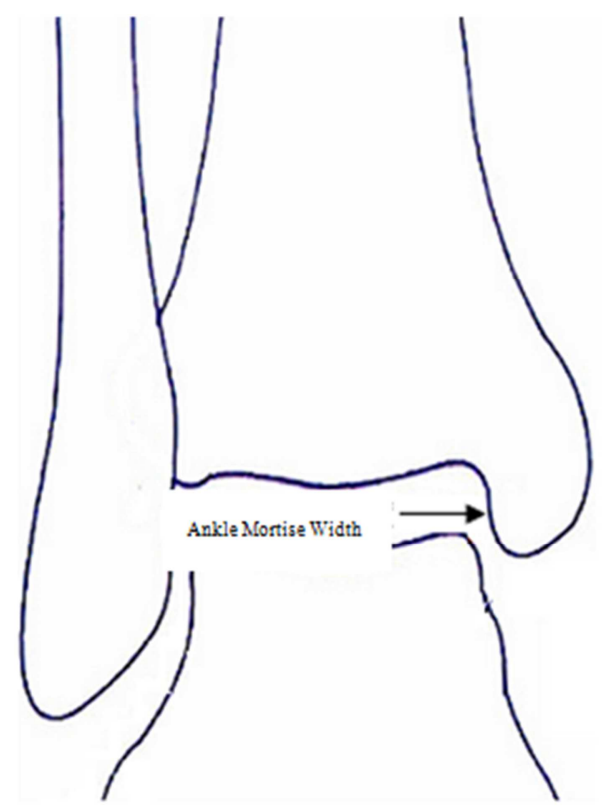

Figure 2. Schematic Diagram for Width of the Ankle Mortise.

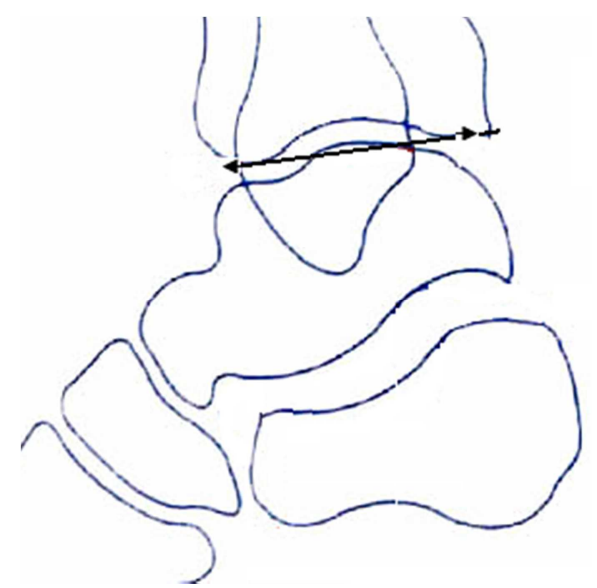

Figure 3. Schematic Diagram for Ankle Mortise Depth (anterior and posterior diameter).

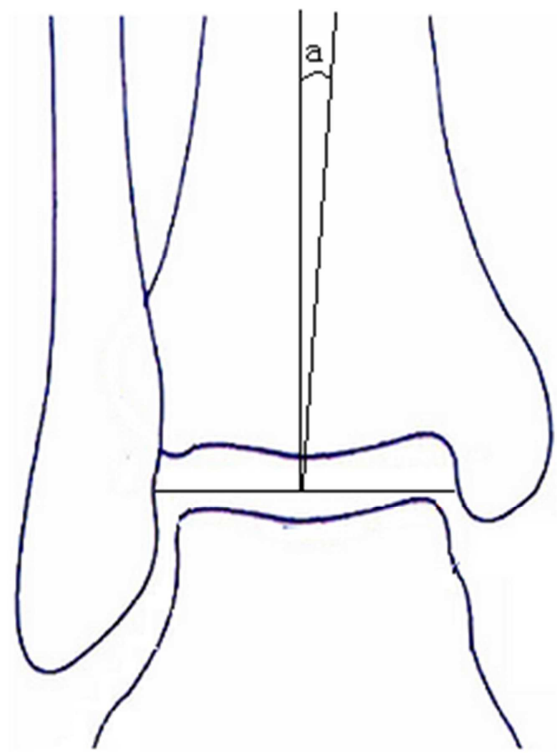

Figure 4. Schematic Diagram for Ankle Mortise Coronal View Angle (a).

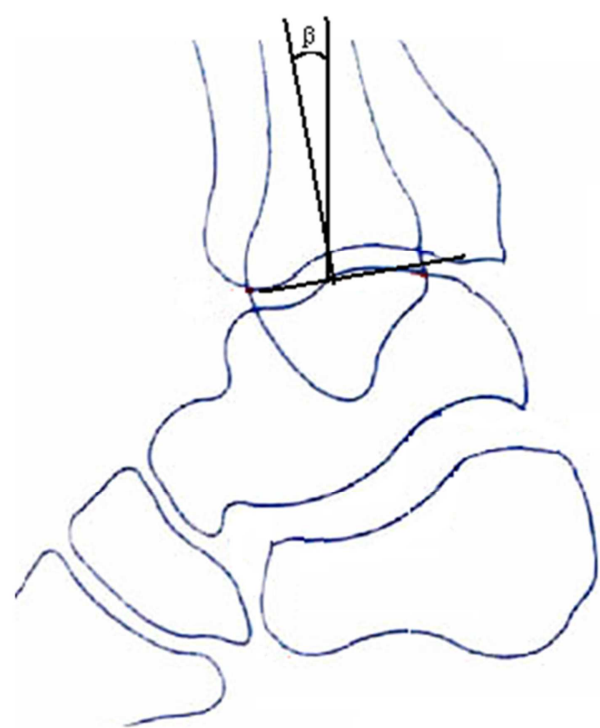

Figure 5. Schematic Diagram for Ankle Mortise Sagittal View Angle ( $\beta$ ).

\subsection{Research Method and Statistical Analysis}

The SPSS 15.0 statistical software was used for data processing. $\mathrm{P}<0.05$ indicates the difference has statistical significance.

(I) Comparison of measurement indexes between the ankle mortise on the unaffected side and affected side: ankle mortise height, width, depth, coronal view angle of the ankle mortise and sagittal view angle of the ankle mortise on unaffected side and affected side of all patients were measured on the X-ray film at the last follow-up visit. Various indexes for unaffected side and affected side were compared using the independent sample $\mathrm{t}$ test.

(II) Comparison of the difference values of ankle mortise measurement indexes for the unaffected side and affected side among the excellent group, good group, moderate+poor group: the difference values of ankle mortise height, width, depth, coronal view angle of the ankle mortise and sagittal view angle of the ankle mortise on unaffected side and affected side of all patients were measured on the X-ray film at the last follow-up visit. A comparison was conducted for the difference values of various indexes for ankle mortises on the affected side and unaffected side among the excellent group, good group, moderate+poor group by a variance analysis.

\section{Results}

\subsection{Adverse Reactions}

In the group, cutaneous necrosis of incision edge occurred to 1 patient with open fracture and was repaired through transfer of a local skin flap. And all other wounds healed by first intention. The time for fracture reduction and fixation for patients of the group was 2-3h averaged at 2.16h. In the group, 3 patients scored as "moderate" and 2 patients scored as "poor" based on scoring system of Mazur et al. suffered from ankylosis 1-3 month (s) after operation and the condition was not significantly improved even though active and passive 
joint exercise treatment was adopted; 1 patient scored as "poor" based on scoring system of Mazur et al. suffered from traumatic arthritis 3-6 months after operation and the long-term follow-up visit showed signs of traumatic arthritis exacerbation even though such treatments as medicine, enhanced muscular strength exercise, mobility aid etc.

\subsection{Comparison Between Measurement Indexes for Ankle Mortises on the Affected Side and Unaffected Side}

The result has shown that based on the comparison between heights of ankle mortises on the affected side and unaffected side the difference has no statistical significance $(\mathrm{P}>0.05)$ indicating that the postoperative height of the ankle mortise on the affected side recovers well and is not significantly different from the normal ankle mortise. Based on the comparison among ankle mortise width, depth, ankle mortise coronal view angle (a) and sagittal view angle $(\beta)$ on the affected side and unaffected side, all differences have statistical significance $(\mathrm{P}<0$. 05) indicating that ankle mortise width, depth, ankle mortise coronal view angle and sagittal view angle change after operation; certain correlation exists between the changes and functions of the ankle joint on the affected side. See Table 1 .

Table 1. Comparison among Ankle Mortise Width, Depth, Ankle Mortise Coronal View Angle (a) and Sagittal View Angle ( $\beta$ ) of the Affected Side and Unaffected Side.

\begin{tabular}{llllll}
\hline Item & Height $(\mathbf{m m})$ & Width $(\mathbf{m m})$ & Depth $(\mathbf{m m})$ & Coronal View Angle $\left({ }^{\circ}\right)$ & Sagittal View Angle $\left({ }^{\circ}\right)$ \\
\hline Affected Side & $31.28 \pm 3.29$ & $40.51 \pm 3.86$ & $42.62 \pm 3.74$ & $9.12 \pm 1.68$ & $16.24 \pm 3.12$ \\
Unaffected side & $30.66 \pm 3.07$ & $36.25 \pm 3.57$ & $37.15 \pm 2.96$ & $6.04 \pm 1.17$ & $11.28 \pm 2.81$ \\
P Value & 0.816 & 0.031 & 0.028 & 0.012 & 0.009 \\
\hline
\end{tabular}

\subsection{Comparison of Difference Values of Measurement Indexes for Ankle Mortises on the Affected Side and Unaffected Side Among the Excellent Group, Good Group, and Moderate+Poor Group}

The statistical analysis results have shown that the difference has no statistical significance based on comparison of height difference values among the three groups $(\mathrm{P}>0.05$, Table 2), indicating that there is no significant correlation between postoperative ankle joint recovery and ankle mortise height. Based on the comparison of width difference values among the three groups, the difference has statistical significance $(\mathrm{P}<0.05$, Table 2$)$. A q test was conducted further for pairwise comparison and the result has shown that all differences based on pairwise comparison of width difference values among the three groups have statistical significance
$(\mathrm{P}<0.05$, Table 3$)$. The average difference value of ankle mortise width in the moderate+poor group $>2 \mathrm{~mm}$ while the average difference value in the excellent group and good group $<1 \mathrm{~mm}$, indicating intraoperative ankle mortise width imposes significant effect on post-operative curative effect. In addition, based on the comparison of difference values of ankle mortise depth, coronal view angle (a) and sagittal view angle $(\beta)$ among the three groups, all differences have statistical significance $(\mathrm{P}<0.05$, Table 2$)$. A $q$ test was conducted further for pairwise comparison and the result has shown that all differences based on pairwise comparison of difference values of depth, coronal view angle and sagittal view angle among the three groups have statistical significance $(\mathrm{P}<0.05$, Table 3$)$ indicating that ankle mortise depth, coronal view angle (a) and sagittal view angle $(\beta)$ impose significant effect on post-operative curative effect.

Table 2. Comparison of Difference Values of Measurement Indexes for Ankle Mortises on the Affected Side and Unaffected Side among the Good Group and Moderate+Poor Group.

\begin{tabular}{lllllll}
\hline Item & Number $(\mathbf{n})$ & $\begin{array}{l}\text { Difference Value of } \\
\text { Height }(\mathbf{m m})\end{array}$ & $\begin{array}{l}\text { Difference Value of } \\
\text { Width (mm) }\end{array}$ & $\begin{array}{l}\text { Difference Value of Difference Value of } \\
\text { Depth }(\mathbf{m m})\end{array}$ & $\begin{array}{l}\text { Difference Value } \\
\text { Coronal View Angle } \\
\text { of Sagittal View } \\
\text { Angle }\left({ }^{\circ}\right)\end{array}$ \\
\hline the Excellent Group & 41 & $0.32 \pm 0.08$ & $0.72 \pm 0.11$ & $1.63 \pm 0.28$ & $2.06 \pm 0.47$ \\
the Good Group & 12 & $0.37 \pm 0.09$ & $1.12 \pm 0.14$ & $2.01 \pm 0.32$ & $2.72 \pm 0.56$ \\
the Moderate+Poor Group & 7 & $0.39 \pm 0.09$ & $2.91 \pm 0.36$ & $3.82 \pm 0.44$ & $4.98 \pm 0.71$ \\
P Value & & 0.715 & 0.007 & 0.016 & 0.012 & $0.95 \pm 0.31$ \\
\hline
\end{tabular}

Table 3. $Q$ Value and P Value based on Pairwise Comparison of Ankle Mortise Width, Depth, Angles of Coronal View and Sagittal View on the Affected Side and Unaffected Side.

\begin{tabular}{|c|c|c|c|c|c|c|c|c|}
\hline \multirow[t]{2}{*}{ Item } & \multicolumn{2}{|c|}{$\begin{array}{l}\text { Difference Value of Width } \\
(\mathrm{mm})\end{array}$} & \multicolumn{2}{|c|}{$\begin{array}{l}\text { Difference Value of Depth } \\
(\mathrm{mm})\end{array}$} & \multicolumn{2}{|c|}{$\begin{array}{l}\text { Difference Value of Coronal } \\
\text { View Angle }\end{array}$} & \multicolumn{2}{|c|}{$\begin{array}{l}\text { Difference Value of Coronal } \\
\text { View Angle }\end{array}$} \\
\hline & q Value & P Value & q Value & P Value & q Value & P Value & q Value & P Value \\
\hline $\begin{array}{l}\text { the Excellent Group: the } \\
\text { Good Group }\end{array}$ & 2.662 & 0.013 & 6.151 & 0.005 & 6.383 & 0.004 & 3.158 & 0.010 \\
\hline $\begin{array}{l}\text { the Good Group: } \\
\text { Moderate+Poor Group }\end{array}$ & 4.256 & 0.008 & 6.843 & 0.003 & 8.651 & 0.002 & 4.565 & 0.007 \\
\hline $\begin{array}{l}\text { the Excellent Group: } \\
\text { Moderate+Poor Group }\end{array}$ & 8.846 & 0.000 & 6.455 & 0.004 & 8.697 & 0.001 & 8.554 & 0.002 \\
\hline
\end{tabular}




\section{Discussion}

Maintenance of the ankle mortise height depends on fibula completeness and recovery of tibia fornix apex. Causes of changes include: (1) fibula or lateral malleolus comminuted fracture displacement causes fibula shortening and extorsion; (2) failure to retract during reduction of distal tibia comminuted fracture; (3) insufficient or no bone grafting at the metaphysis [7-8]. The research results show that the variation range of the ankle mortise height is small, variation range $<0.5 \mathrm{~mm}$ compared with the unaffected side. No significant statistical difference was observed between the height variation and scores of ankle joint functions. We think that the loss of ankle mortise height of patients of the group is a little, which relates to anatomical reduction of fibula and bone graft filling at the metaphysis.

Increased ankle mortise width dislocates astragalus outward in the ankle mortise leading to an unstable ankle joint side, loss of mutual anastomosis on articular surface, shrunken weigh-bearing area, uneven bearing pressure and exacerbation of ischemia and cartilage degeneration [9]. In addition, after the ankle mortise is widened the restrictive effect of the ankle mortise on astragalus weakens and causes instability and traumatic arthritis of ankle joint [10]. The causes of changes in ankle mortise width include: (1) internal malleolus fracture, fibula or unsatisfactory reduction of lateral malleolus fracture displacement; (2) No reduction fixation after distal tibiofibular syndesmosis separation; (3) failure to retract during reduction after transverse separation for the compression bursting fracture at the extremitas anterior of the tibia [11-12]. In the group, the result of the follow-up visit 2 years after the surgery shows that scores of the ankle joints of 7 patients in the moderate+poor group $<80$ points based on the scoring system of Mazur et al. The functions of the ankle joint are poor and the width of the ankle mortise increases more than $2 \mathrm{~mm}$ compared with that on the unaffected side. It indicates that the change of the ankle mortise width impose significant effect on subsequent functions of the ankle joint.

The depth of the ankle mortise increases and makes the astragalus deeply entrapped. Impact is more likely to occur and affects the joint motion [13]. Hak et al. have demonstrated through a biomechanics experiment that posterior malleolus fracture can cause the center of contact stress of ankle joint to move forward and inward and cause the ankle joint to bear huge contact stress thus leading to traumatic arthritis of the ankle joint [14]. Zhang et al thought that the rear fracture block is required to be reduced and fixed by surgery when the distal tibial fracture block is larger than or equal to $10 \%$ of the articular surface of tibial fracture [15]. The causes of change in ankle mortise depth include: (1) bursting fracture at the extremitas inferior of the tibia made the fracture block separated on the sagittal plane and anatomical reduction and fixation were not performed during treatment; (2) the rear bone block at the distal extremity of the tibia was dislocated and difficult to be exposed. The fixation was unsatisfactory [16]. In the group, for patients whose functions of ankle joint scored as moderate+poor, the difference value of the ankle mortises on the affected side and unaffected side is between 2 and $5 \mathrm{~mm} .1 / 2$ osteotomy surface at the extremitas inferior of the tibia is equivalent to $65 \%$ of the normal contact area.

The causes of change in ankle mortise angles include: (1) unsatisfactory reduction of anterolateral bone block (Cha-put node) at extremitas inferior of the tibia; (2) uneven filling for bone graft at the distal extremity of the tibia; (3) improper steel plate and uneven support; (4) too early weight-bearing of the ankle joint [17]. Anatomically, variation in ankle mortise angles reflects the change of weight-bearing force line of the ankle joint [18]. In the group, the coronal view angle of the ankle mortise on the unaffected side is $6.04 \pm 1.17$ and the sagittal view angle is $11.28 \pm 2.81$, which are the normal weight-bearing force line of the ankle joint. Displacement fracture will occur to the fibula and the perpendicular line to the connected line between the two fornix apexes of the ankle mortise and the connected line between the anterior and posterior malleolus rims make approximately an angle of $90^{\circ}$ when the distal extremity of the tibia is damaged due to High-energy injury injury. Thus, coronal view and sagittal view angles of the ankle mortise change. Deviation of the weight-bearing line of the ankle joint will change the distribution of the ankle joint stress leading to osteoarticular load disorder and ankle joint arthritis. The research shows that the difference values of the coronal view angle and sagittal view angle of the ankle mortise have statistical difference among the three groups. During our clinical follow-up visit, traumatic arthritis occurred to the 2 patients who treated with external fixation and who ankle joints were scored $<87$ points based on Mazur et al. scoring system. The difference value between coronal view angles of ankle mortises on the affected side and unaffected side $>5^{\circ}$ and the difference value between sagittal view angles $>3^{\circ}$ indicating that variation in ankle mortise angles affects the functions of ankle joints.

To re-establish an anatomic relation for ankle mortise, firstly, the length of fibula or lateral malleolus should be restored and the fracture extremity stabilized preliminarily; then anatomical reduction will be performed for the articular surface of tibial spur with the three major fracture blocks as the reference points, i.e. internal malleolus fracture block, anterolateral fracture block (Tillaux-Cha-put), posterior lip fracture block (Volkmanns triangle) [19]. The main points for ankle mortise reconstruction by surgery include: (1) restore the weight-bearing line and strength of the tibia and fibula (particularly the tibia), i.e. restore the height of the ankle mortise and bring the coronal view and sagittal view angles of the ankle mortise back to normal; (2) restore the anatomical structure of the articular surface of the tibia and make the articular surface smooth and flat; (3) restore the anatomic relation of the distal tibiofibular syndesmosis; prevent ankle mortise broadening; (4) achieve firm and stable fixation as much as possible to facilitate early mobilization and prevent postoperative ankle mortise deformation [20-21]. A proper medial plate and lateral plate should be selected or addition fixation should be performed for the anterior and posterior 
ends based on the conditions of fracture. Importance should be attached to bone graft for bone defect at the metaphysis so as to ensure that the upper part of the ankle mortise is stable, the reconstructed articular surface is smooth and complete, and the ankle mortise angle is normal.

In conclusion, during treatment of pilon fracture, the image data should be analysed carefully before operation to define fracture classification and degree of injury and master the conditions of fracture block displacement; the reduction principles for pilon fracture should be followed, attention should be paid to bone graft and appropriate and secure fixation mode should be selected to ensure autonomic reduction of ankle mortise morphology during operation; the functions of the ankle joint should be restored as much as possible after operation by early motion and later weight bearing in accordance with the rehabilitation principles for fracture in the vicinity of the joint.

\section{References}

[1] Klammer G. Kadakia AR. et al. Posterior pilon fractures: a retrospective case series and proposed classification system $[\mathrm{J}]$. Foot Ankle Int, 2013, 34 (2): 189-99.

[2] Jansen H. Fenwick A. et al. Clinical outcome and changes in gait pattern after pilon fractures [J]. Int Orthop, 2013, 37 (1): $51-8$.

[3] Gulabi D. Toprak O. et al. The mid-term results of treatment for tibial pilon fractures [J]. Ulus Travma Acil Cerrahi Derg, 2012, 18 (5): 429-35.

[4] Shen QJ. Liu YB. et al. Analyses of relevant influencing factors in the treatment of tibial pilon fractures [J]. Zhonghua Yi Xue Za Zhi, 2012, 92 (27): 1909-12.

[5] Webb MS. Bansal P. Closed reduction of Pilon fractures using an ankle distractor to allow for minimally invasive fixation [J]. Ann R Coll Surg Engl, 2012, 94 (8): 606.

[6] Dresing K. Minimally invasive osteosynthesis of pilon fractures [J]. Oper Orthop Traumatol, 2012, 24 (4): 368-82.

[7] Puha B. Veliceasa B. et al. Minimally invasive percutaneous screws osteosynthesis indications in tibial pilon fractures $[\mathrm{J}]$. Rev Med Chir Soc Med Nat Iasi, 2012, 116 (2): 532-5.

[8] Kiene J. Herzog J. et al. Multifragmentary tibial pilon fractures: midterm results after osteosynthesis with external fixation and multiple lag screws [J]. Open Orthop J, 2012, 6: 419-23.
[9] Tang X. Tang PF. et al. Pilon fractures: a new classification and therapeutic strategies [J]. Chin Med J (Engl), 2012, 125 (14): 2487-92.

[10] Sedlar M. Chrz K. et al. Pilon fractures of the tibia--a short summary of problems and case reports [J]. Rozhl Chir, 2012, 91 (5): 271-5.

[11] Mandi DM. Belin RP. et al. Pilon fractures [J]. Clin Podiatr Med Surg, 2012, 29 (2): 243-78.

[12] Liporace FA. Yoon RS. et al. Decisions and staging leading to definitive open management of pilon fractures: where have we come from and where are we now? [J]. J Orthop Trauma, 2012, 26 (8): 488-98.

[13] Liporace FA. Mehta S. et al. Staged treatment and associated complications of pilon fractures [J]. Instr Course Lect, 2012, 61: 53-70.

[14] Hak DJ. Anterolateral approach for tibial pilon fractures [J]. Orthopedics, 2012, 35 (2): 131-3.

[15] Zhang ZD. Ye XY. et al. Case-control study on minimally invasive percutaneous locking compression plate internal fixation for the treatment of type II and III pilon fractures [J]. Zhongguo Gu Shang, 2011, 12 (24): 1010-2.

[16] Davidovitch RI. Elkhechen RJ. et al. Open reduction with internal fixation versus limited internal fixation and external fixation for high grade pilon fractures (OTA type 43C) [J]. Foot Ankle Int, 2011, 32 (10): 955-61.

[17] Liporace FA. Yoon RS. et al. An adjunct to percutaneous plate insertion to obtain optimal sagittal plane alignment in the treatment of pilon fractures [J]. J Foot Ankle Surg, 2012, 51 (2): 275-7.

[18] Poyanli O. Esenkaya I. et al. Minimally invasive reduction technique in split depression type tibial pilon fractures $[\mathrm{J}]$. $\mathrm{J}$ Foot Ankle Surg, 2012, 51 (2): 254-7.

[19] Zeng XT. Pang GG. et al. Surgical treatment of open pilon fractures [J]. Orthop Surg, 2011, 3 (1): 45-51.

[20] Crist BD. Khazzam M. et al. Pilon fractures: advances in surgical management [J]. J Am Acad Orthop Surg, 2011, 19 (10): 612-22.

[21] Mauffrey C. Vasario G. et al. Tibial pilon fractures: a review of incidence, diagnosis, treatment, and complications [J]. Acta Orthop Belg, 2011, 77 (10): 432-40. 